\title{
Excitation Kinetics of Impurity Doped Quantum Dot Triggered by Gaussian White Noise
}

\author{
Suvajit Pal, ${ }^{1}$ Sudarson Sekhar Sinha, ${ }^{2}$ Jayanta Ganguly, ${ }^{3}$ and Manas Ghosh ${ }^{4}$ \\ ${ }^{1}$ Department of Chemistry, Hetampur Raj High School, Hetampur, Birbhum, West Bengal 731124, India \\ ${ }^{2}$ Department of Physical Chemistry, Indian Association for the Cultivation of Science, Jadavpur, Kolkata, West Bengal 700032, India \\ ${ }^{3}$ Department of Chemistry, Brahmankhanda Basapara High School, Basapara, Birbhum, West Bengal 731215, India \\ ${ }^{4}$ Department of Chemistry, Physical Chemistry Section, Visva Bharati University, Santiniketan, Birbhum, West Bengal 731 235, India
}

Correspondence should be addressed to Manas Ghosh; pcmg77@rediffmail.com

Received 16 April 2013; Accepted 13 May 2013

Academic Editors: I. Galanakis, A. N. Kocharian, and E. Liarokapis

Copyright (C) 2013 Suvajit Pal et al. This is an open access article distributed under the Creative Commons Attribution License, which permits unrestricted use, distribution, and reproduction in any medium, provided the original work is properly cited.

\begin{abstract}
We investigate the excitation kinetics of a repulsive impurity doped quantum dot initiated by the application of Gaussian white noise. In view of a comprehensive research we have considered both additive and multiplicative noise (in Stratonovich sense). The noise strength and the dopant location have been found to fabricate the said kinetics in a delicate way. Moreover, the influences of additive and multiplicative nature of the noise on the excitation kinetics have been observed to be prominently different. The investigation reveals emergence of maximization and saturation in the excitation kinetics as a result of complex interplay between various parameters that affect the kinetics. The present investigation is believed to provide some useful perceptions of the functioning of mesoscopic systems where noise plays some profound role.
\end{abstract}

\section{Introduction}

Throughout last couple of decades we came across a tremendous surge in theoretical and experimental researches on impurity states of low-dimensional heterostructures [1]. Among these heterostructures, quantum dots (QDs) have been found to take some leading role undoubtedly. The importance of QD originates because of its emergence as the destination in the journey towards miniaturization of semiconductor devices. Furthermore, the properties of doped QDs have made them potential candidates for scientific study and technological applications. With QD, the subtle interplay between new confinement sources and impurity potentials has opened up new areas of research in this field [2]. Under the confinement, the dopant location can tailor the electronic and optical properties of the system [3]. This resulted in a rich literature comprising of theoretical studies on impurity states [4-7] in general, and also on their optoelectronic properties, in particular, for a wide range of semiconductor devices [3, 8-15]. The widespread research trend elegantly explores new physics and indicates profound technological impact simultaneously. As a natural extension of the trend, dynamical aspects of doped QD have also been extensively studied. Out of them researches on excitation of strongly confined electron merit importance because of the crucial role played by the said phenomenon in optoelectronic devices and as lasers. From the perspective of technological applications such excitation further involves optical encoding, multiplexing, and photovoltaic and light emitting devices. The phenomenon also plays some important role in the population transfer among the exciton states in QD [16]. Of late, we have performed some inspections which deal with excitation in doped QDs that have been triggered by some external time-varying field $[17,18]$.

Noise in mesoscopic systems influences the functioning of the device to a great extent. This is because of the pivotal role played by the noise as the size of the electronic system reaches the nanometer scale [19]. In practice, noise appears as the main obstacle in the development of semiconductor heterostructure devices for diverse applications and more often than not it also limits the device performance [20]. Hastas et al. have also showed the importance of noise in 
self-assembled InAs QDs embedded in GaAs [21]. The noise can result externally, or it may be intrinsic. Intrinsic noises generally result owing to changes in impurity configurations [22]. However, low frequency noise is one of the prime requirements in QD heterostructures looking at its potential use in a wide range of optoelectronic devices. These applications include industrial manufacturing, medicine, remote sensing, space communications, and military uses [23]. Such noise measurements are also becoming useful as a nondestructive method for the determination of structural disorders entered during production or operation of the devices $[24,25]$. Since QD suffers from a lot of stress during its growth, lattice defects are generated and they diffuse inside the QD structure. The said low-frequency noise also provides a diagnostic tool for defect related properties of materials and structures [26]. Asriyan et al. have extensively studied the role of low-frequency noise in nonhomogeneously doped semiconductors [24].

In the present paper we have investigated the role of Gaussian white noise, applied to the system externally, on the excitation kinetics of doped QD. We have considered both additive and multiplicative noises for an in-depth investigation. Moreover, nowadays the importance of dopant location in fabricating dot properties are well recognized [13, 15]. The distance dependency of dopant unfolds promising opportunities to engineer QD electron dynamics in doped heterostructures. Particularly, Karabulut, Baskoutas, and their coworkers studied the off-center impurities invoking an accurate numerical method (potential morphing method $(P M M))[13,15]$. We therefore, looking at a comprehensive description of our investigation, have determined the timeaverage excitation rate $\left(\left\langle R_{\mathrm{ex}}\right\rangle\right)$ as a function of dopant location $\left(r_{0}\right)$ and noise strength $(\zeta)$. The various profiles unveil the subtle interplay between the dopant location and the noise strength that sensitively modulates the excitation kinetics.

\section{Method}

The model considers an electron subject to a harmonic confinement potential $V(x, y)$ and a perpendicular magnetic field $B$. The confinement potential assumes the form $V(x, y)=(1 / 2) m^{*} \omega_{0}^{2}\left(x^{2}+y^{2}\right)$, where $\omega_{0}$ is the harmonic confinement frequency, $\omega_{c}=e B / m^{*} c$ being the cyclotron frequency (a measure of magnetic confinement offered by $B)$. In the present work a magnetic field of miliTesla (mT) order has been employed. $m^{*}$ is the effective electronic mass within the lattice of the material to be used. We have taken $m^{*}=0.5 m_{0}$ and set $\hbar=e=m_{0}=a_{0}=1$. This value of $m^{*}$ closely resembles $G e$ quantum dots $\left(m^{*}=0.55\right.$ a.u. $)$. We have used Landau gauge $[A=(B y, 0,0)]$ where $A$ stands for the vector potential. The Hamiltonian in our problem reads

$$
\begin{aligned}
H_{0}^{\prime}= & -\frac{\hbar^{2}}{2 m^{*}}\left(\frac{\partial^{2}}{\partial x^{2}}+\frac{\partial^{2}}{\partial y^{2}}\right)+\frac{1}{2} m^{*} \omega_{0}^{2} x^{2} \\
& +\frac{1}{2} m^{*}\left(\omega_{0}^{2}+\omega_{c}^{2}\right) y^{2}-i \hbar \omega_{c} y \frac{\partial}{\partial x} .
\end{aligned}
$$

Define $\Omega^{2}=\omega_{0}^{2}+\omega_{c}^{2}$ as the effective frequency in the $y$ direction. The model Hamiltonian [cf. (1)] sensibly represents a 2 -d quantum dot with a single carrier electron $[27,28]$. The form of the confinement potential conforms to kind of lateral electrostatic confinement (parabolic) of the electrons in the $x-y$ plane $[4,11,29]$.

In the present problem we have considered that the QD is doped with a repulsive Gaussian impurity [30, 31]. Introducing the impurity potential to the Hamiltonian [cf. (1)], it transforms to

$$
H_{0}\left(x, y, \omega_{c}, \omega_{0}\right)=H_{0}^{\prime}\left(x, y, \omega_{c}, \omega_{0}\right)+V_{\mathrm{imp}}\left(x_{0}, y_{0}\right),
$$

where $V_{\text {imp }}\left(x_{0}, y_{0}\right)=V_{\text {imp }}(0)=V_{0} e^{-\gamma_{0}\left[\left(x-x_{0}\right)^{2}+\left(y-y_{0}\right)^{2}\right]}$ with $\gamma_{0}>0$ and $V_{0}>0$ for repulsive impurity, and $\left(x_{0}, y_{0}\right)$ denotes the coordinate of the impurity center. $V_{0}$ is a measure of the strength of impurity potential whereas $\gamma_{0}^{-1}$ determines the spatial stretch of the impurity potential. The presence of repulsive scatterer simulates dopant with excess electrons. The use of such Gaussian impurity potential is quite wellknown [32-34]. The time-independent Schrödinger equation has been solved using variational method expressing the trial wave function $\psi(x, y)$ as a superposition of the product of harmonic oscillator eigenfunctions $[17,18]$. In the linear variational calculation, an appreciably large number of basis functions have been exploited after making the required convergence test.

With the application of external noise the timedependent Hamiltonian reads

$$
H(t)=H_{0}+V(t) .
$$

The noise consists of random term $(\xi(t))$ quite often assumed to be following a Gaussian distribution and characterized by the following equations:

$$
\langle\xi(t)\rangle=0,
$$

the zero mean value condition, and

$$
\left\langle\xi(t) \xi\left(t^{\prime}\right)\right\rangle=2 \zeta \delta\left(t-t^{\prime}\right)
$$

the two-time correlation condition with a negligible correlation time, $\zeta$ being the noise strength. The highly fluctuating term $\xi(t)$ is called white noise because of a flat spectrum in frequency space, like that of white light. The noise strength $\zeta$ becomes simply a measure of intensity of fluctuation. We have invoked Box-Muller algorithm to generate $\xi(t)$. An additive noise term is a random term that does not depend on coordinates of system so that $V(t)=\xi(t)$, whereas a multiplicative noise gets coupled with the system coordinates making $V(t)=\xi(t)(x+y)$ in Stratonovich sense [35].

The time-dependent Schrödinger equation (TDSE) containing the evolving wave function has now been solved numerically by 6 th order Runge-Kutta-Fehlberg method. In the numerical solution appropriate initial conditions have been used and numerical stability has been checked. We define the quantity $P_{k}(t)=\left|a_{k}(t)\right|^{2}$ to indicate the population of $k$ th state of $H_{0}$ at time $t$ where $a_{k}(t)$ is the timedependent superposition coefficient for the $k$ th eigenstate 


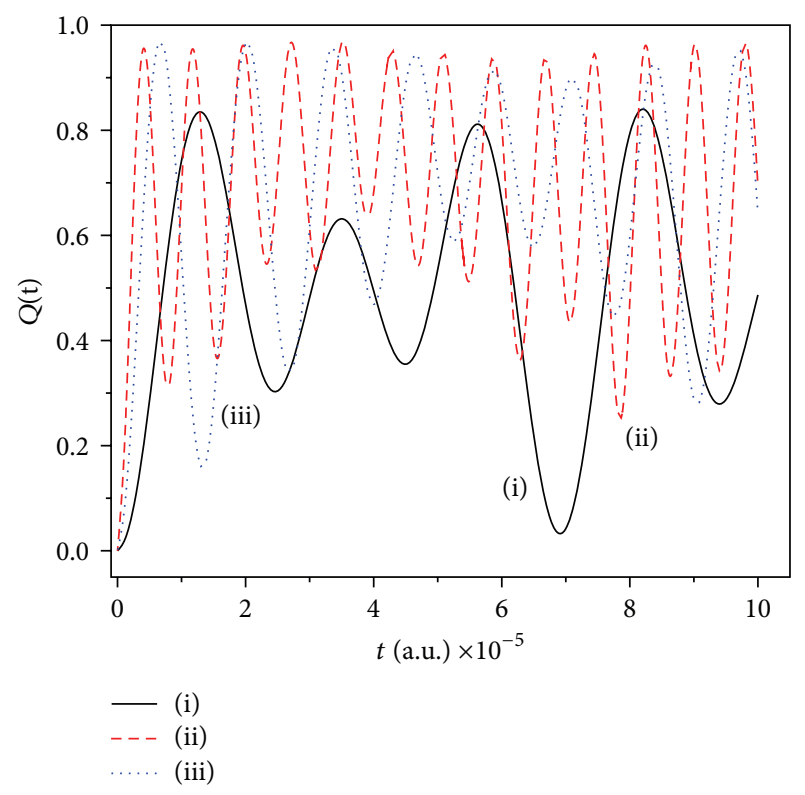

FIGURE 1: Plot of $Q(t)$ versus $t$ for Gaussian additive white noise of fixed strength $\zeta=1.0 \times 10^{-6}$ a.u. at three different dopant locations $\left(r_{0}\right)$ : (i) 0.0 a.u., (ii) 28.28 a.u., and (iii) 70.71 a.u.

of the unperturbed Hamiltonian. We observe a continuous variation in the ground state population $\left[P_{0}(t)\right]$ during the time evolution. Naturally the quantity $Q(t)=1-P_{0}(t)$ serves as a measure of excitation. In consequence, the quantity $R_{\text {ex }}(t)=d Q / d t$ serves as the time-dependent rate of excitation from which the time-average rate of excitation $\left[\left\langle R_{\mathrm{ex}}\right\rangle=(1 / T) \int_{0}^{T} R_{\mathrm{ex}}(t) d t\right]$ can be calculated, where $T$ is the total time of dynamic evolution $[17,18]$.

\section{Results and Discussion}

3.1. Role of Additive Noise. Figure 1 displays the excitation profile of the doped dot as a function of time $(t)$ when an additive noise of fixed strength $\left(\zeta=1.0 \times 10^{-6}\right.$ a.u.) has been applied for three different dopant locations $\left(r_{0}\right)$. The plots reveal lot of undulations at all dopant locations indicating occurrence of considerable amount of excitation and deexcitation in tandem. However, extent of oscillation becomes more vigorous at off-center locations $\left(r_{0}=28.28\right.$ a.u. and 70.71 a.u.) in comparison to the on-center dopant $\left(r_{0}=\right.$ 0.0 a.u.). The variation in the extent of oscillation at onand off-center locations can be attributed to varied influence of dot confinement center at these locations. The reduced strength of dot confinement at off-center locations makes the noise term more prominently active resulting in an enhancement in the oscillations of excitation profile. At oncenter dopant location, because of the strong confinement offered by dot, the noise effect remains submissive to some extent which restricts the oscillation a bit.

In order to analyze the exclusive role played by the dopant location on excitation kinetics, we have plotted $\left\langle R_{\mathrm{ex}}\right\rangle$ as a function of dopant location for a fixed value of noise strength $\left(\zeta=1.0 \times 10^{-6}\right.$ a.u.) (Figure 2). The plot reveals a distinct

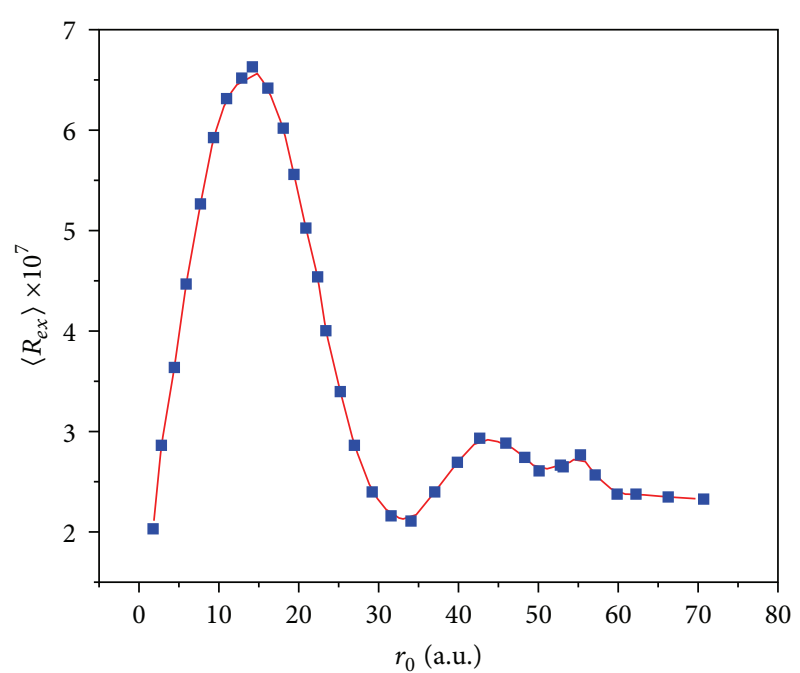

FIGURE 2: Plot of $\left\langle R_{\text {ex }}\right\rangle$ versus $r_{0}$ for additive white noise of fixed strength $\zeta=1.0 \times 10^{-6}$ a.u.

maximization in the rate at $r_{0} \sim 14.0$ a.u. It needs to be realized that the role of dopant coordinate on excitation rate is not at all straightforward, rather it is quite delicate. An increase in $r_{0}$ takes the dopant away from the confinement of dot potential favoring the excitation. However, the said shift also reduces dot-impurity repulsive interaction thereby suppressing excitation. Thus, a progressive shift of dopant to more and more off-center positions invites two opposite factors come into play that can affect excitation rate. It is the changeover of relative dominance of one of the factor over the other that causes maximization in the kinetics at some typical dopant location. At far off-center locations $\left(r_{0} \geq 60.0\right.$ a.u. $)$ we envisage kind of saturation in the kinetics owing to sort of balance between the opposing factors.

Surprisingly enough, in the present case we do not envisage any influence of noise strength $(\zeta)$ on the excitation kinetics. The reluctance of noise strength to influence the excitation is interesting and presumably has origin in its very "additive" nature. It seems that, remaining uncoupled to the system coordinates, the additive noise simply adds on to the Hamiltonian and thus perturbs all the eigenstates to equal extents. Thus, on the whole, the relative separations between all the eigenstates remain unaltered making the excitation kinetics a mute spectator of the noise strength. This observation further necessitates investigation of the role of multiplicative noise in the present context.

3.2. Role of Multiplicative Noise. Figure 3 exhibits the excitation profile induced by a multiplicative white noise for the same fixed value of $\zeta$ at three different dopant locations. Unlike to the case of additive noise, here the extent of oscillation is found to be more or less equivalent at these dopant locations. Thus, insofar as excitation and deexcitation are concerned, coupling the noise term to the system coordinate brings about more subtle interplay between the dot confinement and the noise strength. 


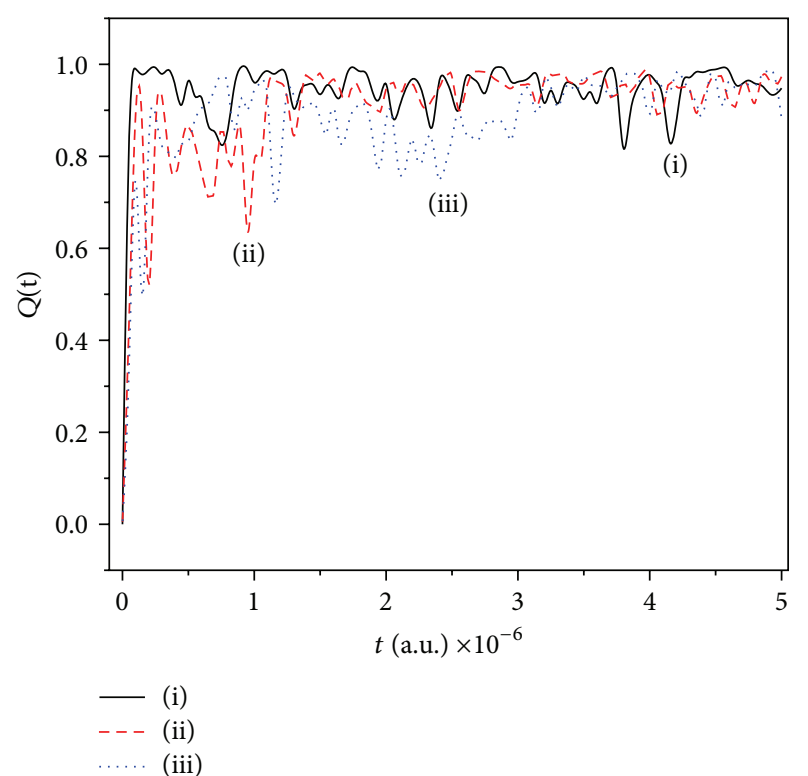

FIgURE 3: Plot of $Q(t)$ versus $t$ for Gaussian multiplicative white noise of fixed strength $\zeta=1.0 \times 10^{-6}$ a.u. at three different dopant locations $\left(r_{0}\right)$ : (i) 0.0 a.u., (ii) 28.28 a.u., and (iii) 70.71 a.u.

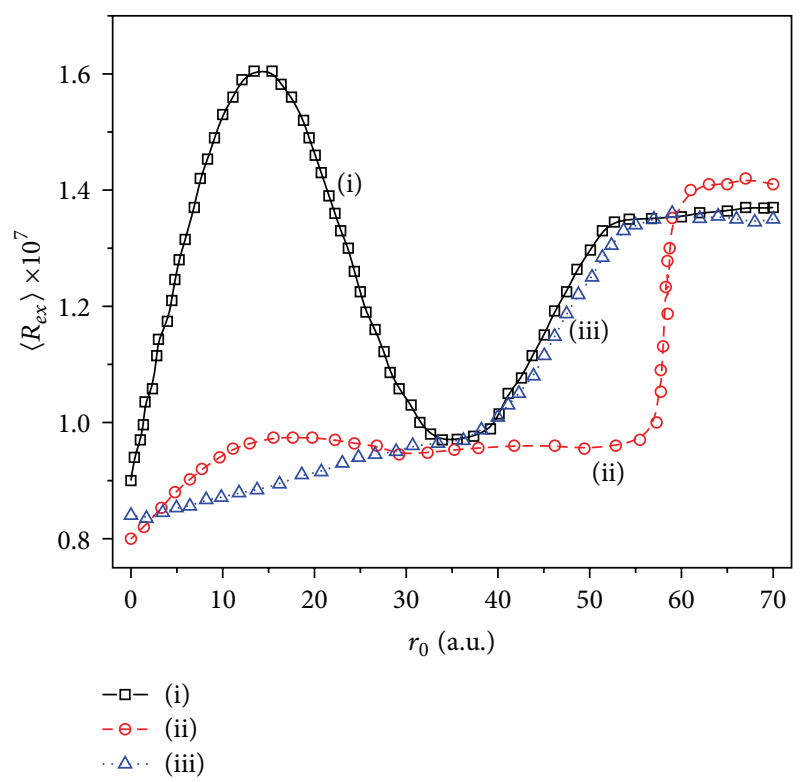

Figure 4: Plot of $\left\langle R_{\text {ex }}\right\rangle$ versus $r_{0}$ for Gaussian multiplicative white noise for different noise strengths $(\zeta)$ : (i) $\zeta=1.0 \times 10^{-7}$ a.u., (ii) $\zeta=1.0 \times 10^{-5}$ a.u., and (iii) $\zeta=1.0 \times 10^{-4}$ a.u.

Figure 4 delineates $\left\langle R_{\mathrm{ex}}\right\rangle$ as a function of dopant location for different values of $\zeta$. Already, in the context of Figure 2, we have discussed how different factors inherently affect the excitation kinetics in contrasting ways as the dopant is shifted to more and more off-center locations. However, in addition to the said factors, now we have to take into account the contribution from $\zeta$ also; by virtue of its intrinsic characteristic the noise is likely to promote excitation. From Figure 4 we notice that at low noise strength $\left(\zeta=1.0 \times 10^{-7}\right.$ a.u. $)$, the excitation rate passes through a maxima at $r_{0} \sim 14.0$ a.u. (nearly at the same dopant location where we have observed maximization in case of additive noise, Figure 2). However, no such maximization is observed using multiplicative noise of medium $(\zeta=1.0 \times$ $10^{-5}$ a.u. $)$ and high $\left(\zeta=1.0 \times 10^{-4}\right.$ a.u. $)$ strength. It is also evident from the figure that for all noise strengths the excitation rate attains steady value at far off-center dopant locations. A multiplicative noise of extremely low strength perturbs the system feebly and hardly leaves its signature on excitation kinetics. Thus, the excitation kinetics effectively depends on the dopant location and exhibits similar pattern as that of additive noise. As the noise strength assumes some intermediate value $\left(\zeta=1.0 \times 10^{-5}\right.$ a.u. $)$, the rate profile after some small initial rise exhibits a steady behavior within the range 15.7 a.u. $\leq r_{0} \leq 55.5$ a.u. Beyond $r_{0} \geq$ 55.5 a.u. the excitation rate jumps abruptly to a high value and permanently settles to steady value beyond $r_{0} \geq 63.1$ a.u. Such rate profile reveals the nuances of combined influence of several factors that affect excitation. The initial small rise in $\left\langle R_{\mathrm{ex}}\right\rangle$ at near off-center locations $\left(r_{0} \leq 15.7\right.$ a.u. $)$ indicates mild win of noise and dot-impurity repulsive terms over that of strong confinement potential. After that there is a sustained balance between the diverse factors that affect excitation over a quite extended range of dopant location (15.7 a.u. $\leq r_{0} \leq 55.5$ a.u.). At $r_{0}=55.5$ a.u., the confinement becomes quite weak and factors that promote excitation (noise + repulsive interaction) suddenly become prominent leading to an abrupt rise in excitation rate. Beyond $r_{0} \geq 63.1$ a.u., because of large dot-impurity separation, their mutual repulsive interaction gets considerably diminished and we land up at a permanent balanced condition of those factors revealed through a saturation in excitation kinetics. With a high value of noise strength $\left(\zeta=1.0 \times 10^{-4}\right.$ a.u. $)$ the rate profile manifests kind of resemblance with that of medium noise strength counterpart. However, the variation in the rate with dopant location appears much less sharp. The initial rise of $\left\langle R_{\mathrm{ex}}\right\rangle$ occurs over an extended region (0.0 a.u. $\leq r_{0} \leq 35.0$ a.u.) in an indolent manner; the former extended zone of constant excitation rate becomes nearly absent and the rate begins to rise as $r_{0} \geq 35.0$ a.u. From the figure it is also quite discernible that the rise in excitation rate within 35.0 a.u. $\leq r_{0} \leq 56.6$ a.u. becomes much less abrupt in comparison with the medium noise strength counterpart. The regime of persistent saturation in excitation kinetics begins from $r_{0}=56.6$ a.u. Thus, an increase in noise strength from medium to high value does not much alter the intrinsic nature of interplay between itself, the dot confinement potential, and the dot-dopant repulsive interaction (the last two factors undergo change with a variation in dopant location) toward fabricating the excitation kinetics. The said increase seems to disseminate the complex interplay over a spatially more spread out domain making the overall variation of the excitation rate rather sluggish with change in dopant coordinate.

In the concluding section of our discussion we would like to demonstrate the variation of $\left\langle R_{\mathrm{ex}}\right\rangle$ with $\zeta$ at on-center 


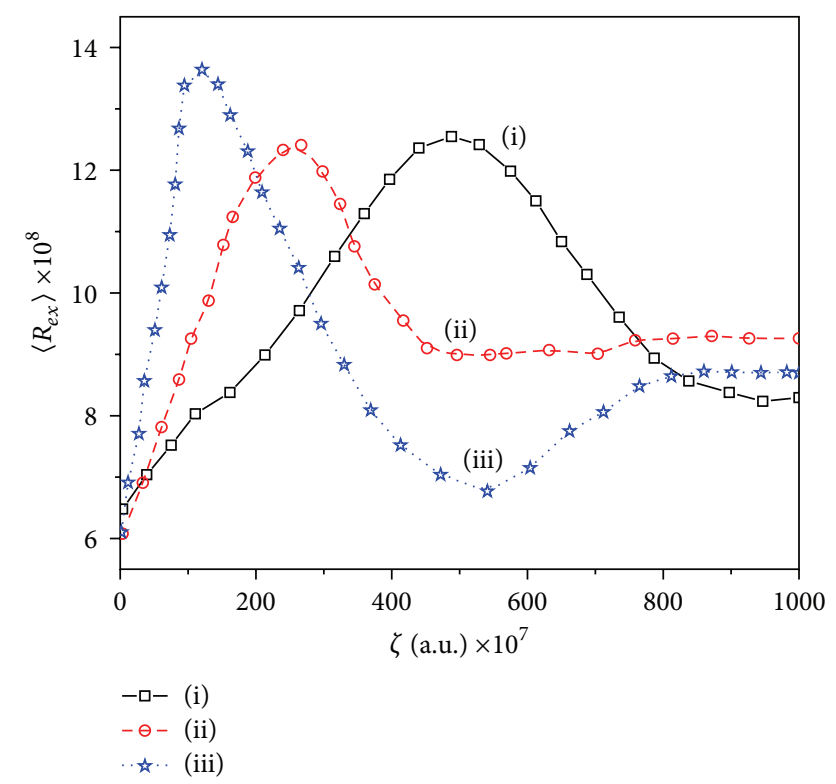

Figure 5: Plot of $\left\langle R_{\mathrm{ex}}\right\rangle$ versus $\zeta$ for Gaussian multiplicative white noise for different dopant locations $\left(r_{0}\right)$ : (i) $r_{0}=0.0$ a.u., (ii) $r_{0}=$ 28.28 a.u., and (iii) $r_{0}=70.71$ a.u.

$\left(r_{0}=0.0\right.$ a.u. $)$ and two off-center $\left(r_{0}=28.28\right.$ a.u. and 70.71 a.u.) dopant locations using multiplicative noise (Figure 5). Such a demonstration focuses mainly on the role played by the noise strength in shaping the excitation kinetics. Figure 5 evinces that, more or less at all dopant locations, $\left\langle R_{\mathrm{ex}}\right\rangle$ passes through a maximum for some typical values of noise strength depending upon the dopant location. Moreover, the kinetics terminates to some saturation at high values of noise strength. The appearance of maxima can as usually be conceived on the basis of changeover of relative dominance of parameters having contrasting influences on excitation kinetics. The changeover is actually associated with the variation of noise strength. It is also interesting to note from the figure that as the dopant is shifted from on to more and more off-center locations the emergence of maxima occurs at lower $\zeta$ values, namely, at $\zeta=4.87 \times 10^{-5}$ a.u. for $r_{0}=0.0$ a.u., $\zeta=2.65 \times 10^{-5}$ a.u. for $r_{0}=28.28$ a.u., and $\zeta=$ $1.17 \times 10^{-5}$ a.u. for $r_{0}=70.70$ a.u. It seems that, since such a shift of the dopant takes it away from dot confinement center, the noise becomes progressively more alacritous in bringing about the changeover of parameters that affect excitation kinetics. The emergence of maxima thus occurs at more and more low values of noise strength.

\section{Conclusions}

The excitation kinetics of impurity doped quantum dots insisted by the Gaussian white noise reveals some interesting aspects. The interplay between the noise strength and the dopant location delicately modulates the kinetics. We envisage a dopant position dependent maximization in the excitation rate in case of additive noise. However, in case of additive noise we have found the noise strength remaining indifferent toward the excitation rate. The interplay between dopant location and noise strength becomes more subtle in case of multiplicative noise. Now excitation rate has been found to display distinct characteristics as dopant location and noise strength are varied over a wide range. Whereas the appearance of maxima in the excitation kinetics indicate preponderance of the parameters that favor excitation over the opposite ones, the saturation in the kinetics can be attributed to kind of compromise between these rival factors. The results are thus quite interesting and expected to convey important insights in related field of research.

\section{Acknowledgments}

The authors Suvajit Pal, Jayanta Ganguly and Manas Ghosh thank Department of Science and Technology FIST (Government of India) and UGC-SAP (Government of India) for partial financial support.

\section{References}

[1] P. M. Koenraad and M. E. Flatté, "Single dopants in semiconductors," Nature Materials, vol. 10, pp. 91-100, 2011.

[2] J. L. Movilla and J. Planelles, "Off-centering of hydrogenic impurities in quantum dots," Physical Review B, vol. 71, Article ID 075319, 7 pages, 2005.

[3] M. J. Kelly, Low-Dimensional Semiconductors, Oxford University Press, Oxford, UK, 1995.

[4] B. Gülveren, Ü. Atav, M. Sahin, and M. Tomak, "A parabolic quantum dot with $\mathrm{N}$ electrons and an impurity," Physica E, vol. 30, pp. 143-149, 2005.

[5] E. Räsänen, J. Könemann, R. J. Puska, M. J. Haug, and R. M. Nieminen, "Impurity effects in quantum dots: toward quantitative modeling," Physical Review B, vol. 70, Article ID 115308, 6 pages, 2004.

[6] M. Aichinger, S. A. Chin, E. Krotscheck, and E. Räsänen, "Effects of geometry and impurities on quantum rings in magnetic fields," Physical Review B, vol. 73, Article ID 195310, 8 pages, 2006.

[7] W. Xie, "Binding energy of an off-center hydrogenic donor in a spherical Gaussian quantum dot," Physica B, vol. 403, no. 17, pp. 2828-2831, 2008.

[8] C. P. Poole Jr. and F. J. Owens, Introduction to Nanotechnology, Wiley, New York, NY, USA, 2003.

[9] W. Xie, "Impurity effects on optical property of a spherical quantum dot in the presence of an electric field," Physica B, vol. 405, no. 16, pp. 3436-3440, 2010.

[10] W. Xie, "Optical properties of an off-center hydrogenic impurity in a spherical quantum dot with Gaussian potential," Superlattices and Microstructures, vol. 48, no. 2, pp. 239-247, 2010.

[11] A. J. Peter, "Polarizabilities of shallow donors in spherical quantum dots with parabolic confinement," Physics Letters A, vol. 355, pp. 59-62, 2006.

[12] K. M. Kumar, A. J. Peter, and C. W. Lee, "Optical properties of a hydrogenic impurity in a confined $Z n_{1-x} C d_{x} S e / Z n S e$ spherical quantum dot," Superlattices and Microstructures, vol. 51, pp. 184193, 2012.

[13] S. Baskoutas, E. Paspalakis, and A. F. Terzis, "Electronic structure and nonlinear optical rectification in a quantum dot: effects 
of impurities and external electric field," Journal of Physics Condensed Matter, vol. 19, no. 39, Article ID 395024, 2007.

[14] I. Karabulut and S. Baskoutas, "Linear and nonlinear optical absorption coefficients and refractive index changes in spherical quantum dots: effects of impurities, electric field, size, and optical intensity," Journal of Applied Physics, vol. 103, no. 7, Article ID 073512, 2008.

[15] I. Karabulut and S. Baskoutas, "Second and third harmonic generation susceptibilities of spherical quantum dots: effects of impurities, electric field and size," Journal of Computational and Theoretical Nanoscience, vol. 6, no. 1, pp. 153-156, 2009.

[16] E. Paspalakis and A. F. Terzis, "Controlled excitonic population transfer in a quantum dot system interacting with an electromagnetic field: local field effects," in Proceedings of the 5th International Conference on Microelectronics, Nanoelectronics and Optoelectronics, pp. 44-49, 2006.

[17] K. Sarkar, N. K. Datta, and M. Ghosh, "Dynamics of electron impurity doped quantum dots in the presence of time-varying fields: influence of impurity location," Physica E, vol. 43, no. 1, pp. 345-353, 2010.

[18] N. K. Datta, D. Konar, and M. Ghosh, "Impurity controlled excitations in doped quantum dot exposed to periodic external perturbation," Microelectronic Engineering, vol. 88, pp. 33063311, 2011.

[19] H. K. Zhao, "Shot noise in the hybrid systems with a quantum dot coupled to normal and superconducting leads," Physics Letters A, vol. 299, pp. 262-270, 2002.

[20] N. A. Hastas, C. A. Dimitriadis, L. Dozsa, E. Gombia, S. Amighetti, and P. Frigeri, "Low frequency noise of GaAs Schottky diodes with embedded InAs quantum layer and selfassembled quantum dots," Journal of Applied Physics, vol. 93, no. 7, pp. 3990-3994, 2003.

[21] N. A. Hastas, C. A. Dimitriadis, L. Dozsa, E. Gombia, and R. Mosca, "Investigation of single electron traps induced by InAs quantum dots embedded in $G a A s$ layer using the low-frequency noise technique," Journal of Applied Physics, vol. 96, no. 10, pp. 5735-5739, 2004.

[22] M. Pioro-Ladrière, J. H. Davies, A. R. Long et al., "Origin of switching noise in GaAs AlxGa1-xAs lateral gated devices," Physical Review B, vol. 72, no. 11, Article ID 115331, 8 pages, 2005.

[23] P. Yuan, O. Baklenov, H. Nie, A. L. Holmes, B. G. Streetman, and J. C. Campbell, "High-speed and low-noise avalanche photodiode operating at $1.06 \mu \mathrm{m}$," IEEE Journal on Selected Topics in Quantum Electronics, vol. 6, no. 3, pp. 422-425, 2000.

[24] H. V. Asriyan, F. V. Gasparyan, V. M. Aroutiounian, S. V. Melkonyan, and P. Soukiassian, "Low-frequency noise in nonhomogeneously doped semiconductor," Sensors and Actuators A, vol. 113, no. 3, pp. 338-343, 2004.

[25] Z. Chabola and A. Ibrahim, "Noise and scanning by local illumination as reliability estimation for silicon solar cells," Fluctuation and Noise Lett, vol. 1, pp. L21-L26, 2001.

[26] J. I. Lee, H. D. Nam, W. J. Choi et al., "Low frequency noise in GaAs structures with embedded In $(\mathrm{Ga})$ As quantum dots," Current Applied Physics, vol. 6, no. 6, pp. 1024-1029, 2006.

[27] L. Jacak, P. Hawrylak, and A. Wojos, Quantum Dots, Springer, Berlin, Germany, 1998.

[28] T. Chakraborty, Quantum Dots-A Survey of the Properties of Artificial Atoms, Elsevier, Amsterdam, The Netherlands, 1999.

[29] S. Baskoutas, A. F. Terzis, and E. Voutsinas, "Binding energy of donor states in a quantum dot with parabolic confinement," Journal of Computational and Theoretical Nanoscience, vol. 1, no. 3, pp. 317-321, 2004.
[30] V. Halonen, P. Hyvönen, P. Pietiläinen, and T. Chakraborty, "Effects of scattering centers on the energy spectrum of a quantum dot," Physical Review B, vol. 53, pp. 6971-6974, 1996.

[31] V. Halonen, P. Pietilinen, and T. Chakraborty, "Opticalabsorption spectra of quantum dots and rings with a repulsive scattering centre," Europhysics Letters, vol. 33, pp. 337-382, 1996.

[32] J. Adamowski, A. Kwásniowski, and B. Szafran, "LO-phononinduced screening of electronelectron interaction in $D^{-}$centres and quantum dots," Journal of Physics, vol. 17, pp. 4489-4500, 2005.

[33] S. Bednarek, B. Szafran, K. Lis, and J. Adamowski, "Modeling of electronic properties of electrostatic quantum dots," Physical Review B, vol. 68, Article ID 155333, 9 pages, 2003.

[34] B. Szafran, S. Bednarek, and J. Adamowski, "Parity symmetry and energy spectrum of excitons in coupled self-assembled quantum dots," Physical Review B, vol. 64, Article ID 125301, 10 pages, 2001.

[35] J. García-Ojalvo and J. M. Sancho, Noise in Spatially Extended Syatems, Springer, New York, NY, USA, 1999. 

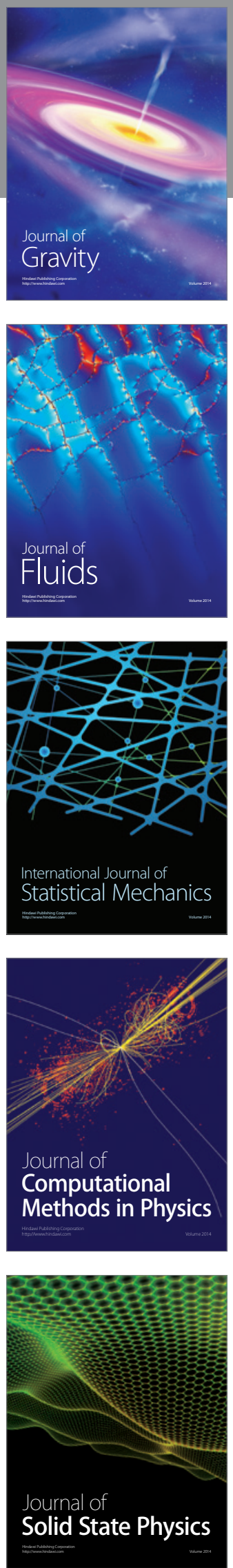

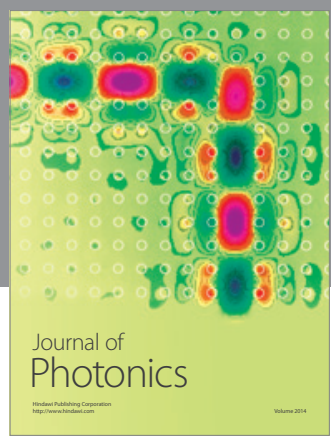

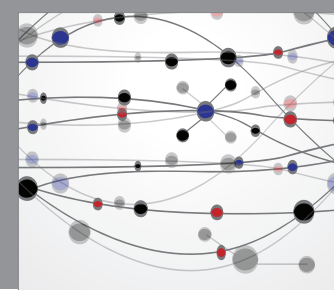

The Scientific World Journal

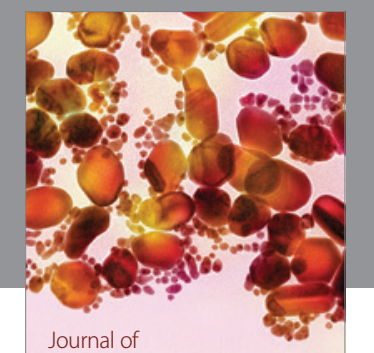

Soft Matter
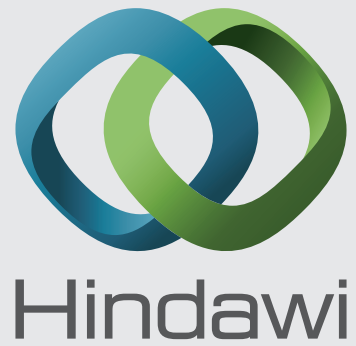

Submit your manuscripts at

http://www.hindawi.com
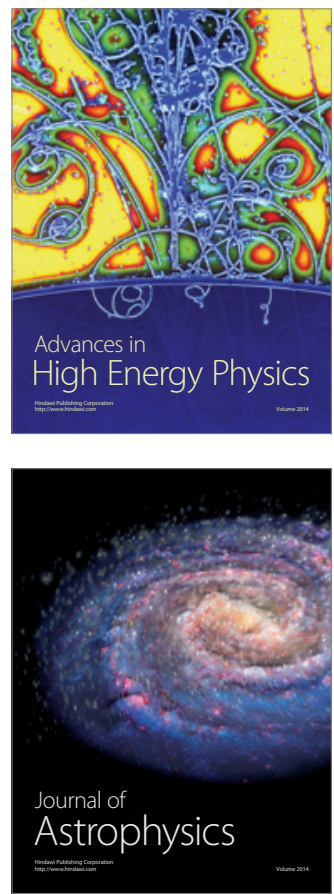
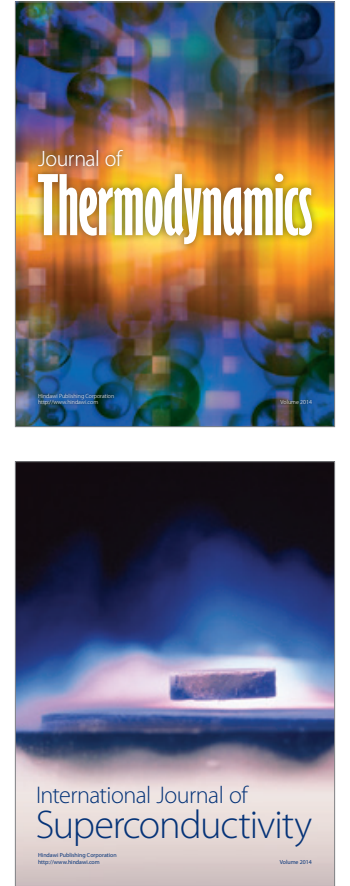
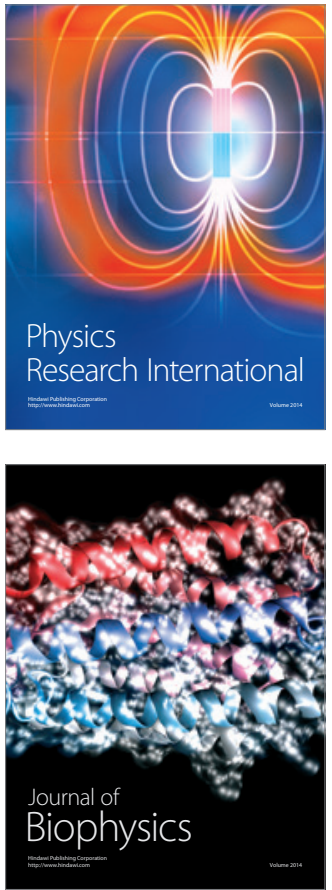
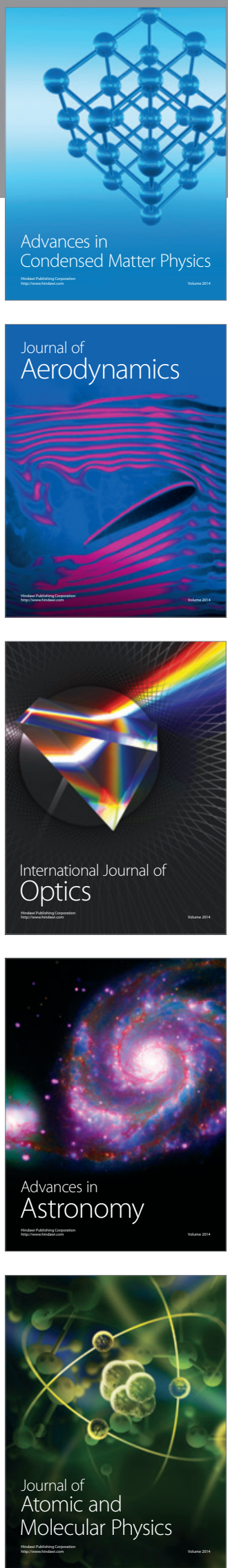